\title{
Synergistic effect of interleukin- 6 and endoplasmic reticulum stress inducers on the high level of ABCG2 expression in plasma cells
}

\author{
Naoto Nakamichi ${ }^{1}{ }^{2}$, Eiichi Morii ${ }^{1}$, Jun-ichiro Ikeda ${ }^{1}$, Ying Qiu ${ }^{1}$, Suhana Mamato ${ }^{1}$, Tian Tian ${ }^{1}$, Shirou Fukuhara ${ }^{2}$ \\ and Katsuyuki Aozasa ${ }^{1}$
}

\begin{abstract}
ABCG2 is a transporter preferentially expressed in a primitive subpopulation of cells and recently reported as a surviving factor for trophoblasts. To date, manner of ABCG2 expression in lymphoid tissues is not known. Immunohistochemically, strong $A B C G 2$ expression was found in a small proportion of plasma cells mainly located in the interfollicular space of lymphoid tissues. The number of ABCG2-high plasma cells increased in interleukin-6- (IL-6) rich lesions, such as Castleman's disease of plasma cell type. Plasma cells are subjected to endoplasmic reticulum (ER) stress when excess proteins are synthesized, and IL- 6 stimulates protein synthesis. Therefore, the effect of IL- 6 and ER stress on ABCG2 expression in plasma cells was examined. The expression level of ABCG2 increased by treatment with either IL- 6 or ER stress inducers, and further increased with both. The promoter analysis revealed that the effect of IL-6 and ER stress inducers was mediated through the site overlapping XBP-1 and HIF-1 binding sequences. Knocked-down of ABCG2 by siRNA or ABCG2 inhibitor reduced plasma cell viability under ER stress. These suggest that $A B C G 2$ is a surviving factor for plasma cells. To our knowledge, this is the first study reporting the effect of ER stress on ABCG2 expression.
\end{abstract}

Laboratory Investigation (2009) 89, 327-336; doi:10.1038/labinvest.2008.157; published online 12 January 2009

KEYWORDS: ABCG2; ER stress; immunohistochemistry; lymph nodes; plasma cells

The family of adenosine triphosphate binding cassette $(\mathrm{ABC})$ transporters mediates the transfer of substrates across the cellular membrane. ${ }^{1,2}$ Among ABC transporters, ABCG2, also called breast cancer-resistant protein, is expressed in a more primitive subpopulation of cells, such as hematopoietic, embryonic, and mesenchymal stem cells. ${ }^{3}$ When cells are stained with Hoechst 33342 dye, a small population of cells, called side population, is known to expel dye efficiently. ${ }^{4}$ ABCG2 is reported to be involved in this process, therefore the side population is supposed to contain stem cells. ${ }^{5,6}$ Among human hematopoietic cells, the CD34-positive, CD38-negative, and lineage-negative stem cell population expresses ABCG2 at the highest level. ${ }^{5,7}$ The CD34-negative, $\mathrm{CD} 7$-positive, and lineage-negative population containing lymphoid progenitor cells also express ABCG2.$^{8,9}$

In addition to the transport of a broad range of xenobiotics, ABCG2 is involved in protection of cells from endogenous and exogenous stress factors, including proinflammatory mediators and hypoxia. ${ }^{10}$ Recently, Evseenko et $\mathrm{al}^{11}$ reported that reduced expression of ABCG2 resulted in idiopathic human fetal growth restriction, thus suggested that ABCG2 is a placental survival factor. Although the mechanisms have not been elucidated, ABCG2 may contribute to the maintenance of stem or progenitor cells as a survival factor.

Immunohistochemical studies have revealed that ABCG2 is expressed in the plancental syncytiotrophoblasts, bile canaliculi of the liver, epithelium of intestine and prostate, and blood vessels. ${ }^{12,13}$ Although, ABCG2 expression in lymphoid tissues is unknown. In this study, ABCG2 expression in lymphoid tissues under various pathological stimuli was examined. We found significantly strong ABCG2 expression in a small proportion of plasma cells, which are known to be under the endoplasmic reticulum (ER)-stressed condition for synthesis of a large amount of proteins. ${ }^{14}$ It is reported that hypoxia induces increase of ABCG2 expression level, ${ }^{15}$ but

\footnotetext{
Department of Pathology, Graduate School of Medicine, Osaka University, Osaka, Japan and ²First Department of Internal Medicine, Kansai Medical University, Osaka, Japan

Correspondence: Dr E Morii, MD, Department of Pathology, Graduate School of Medicine, Osaka University, Yamada-oka 2-2, Suita 565-0871, Japan.

E-mail: morii@patho.med.osaka-u.ac.jp

Received 30 April 2008; revised 08 October 2008; accepted 29 October 2008
} 
effect of ER stress on ABCG2 expression remains unclear. We hypothesized that a small population of plasma cells might express high level of ABCG2 under severe ER stress. In fact, the expression level of ABCG2 increased in plasma cell lines under severe ER stress. The high level of ABCG2 expression in plasma cells suggests that ABCG2 work as a surviving factor in plasma cells under ER stress.

\section{MATERIALS AND METHODS Cells and Reagents}

The human plasma cell lines RPMI8226 and U266, and human B lymphoblastoid cell line Skw6.4 were kindly provided by Dr K Ishihara (Kawasaki Medical School, Kurashiki, Japan). Cells were cultured in RPMI1640 medium (Sigma, St. Louis, MO, USA) supplemented with $10 \%$ fetal calf serum (FCS; Nippon Bio-supp. Center, Tokyo, Japan). Tunicamycin, thapsigargin, 2-deoxyglucose, 5-aza-2'-deoxycitidine (5-aza dC) were purchased from Sigma, recombinant human interleukin-6 (IL-6) from R\&D Systems (Minneapolis, MN, USA), and ABCG2 inhibitor fumitremorgin C (FTC) from Alexis Corp (San Diego, CA, USA), respectively.

\section{Patients and Immunohistochemistry}

Clinical samples of lymph nodes and tonsils resected at Osaka University Hospital were fixed in $10 \%$ formalin, routinely processed for paraffin embedding, and stored in the dark room in the Department of Pathology of Osaka University Hospital at room temperature. They included 33 cases of reactive lymphoid hyperplasia (lymphoid follicular hyperplasia of tonsil-chronic tonsillitis 13 cases and lymph nodes 9 cases) and Castleman's disease, plasma cell type (11 cases). A total of 91 cases of lymphomas were also examined; diffuse large B-cell lymphoma (DLBCL, 20 cases), follicular lymphoma (FL, 19 cases), Hodgkin lymphoma (HL, 15 cases), peripheral T-cell lymphoma unspecified (PTCL-u, 12 cases), and angioimmunoblastic T-cell lymphoma (AILT, 25 cases). Histologic sections cut at $4 \mu \mathrm{m}$ were stained with hematoxylin and eosin, and immunoperoxidase procedure (avidin-biotin complex method). By immunohistochemical procedure, histologic sections were treated with Pascal-pressurized heating chamber (DAKO A/S, Glostrup, Denmark) for antigen retrieval, incubated with anti-ABCG2 antibody (clone BXP-21; Kamiya Biomedical Company, Tukwila, WA, USA), anti-CD138 (Santa Cruz Biotech, Santa Cruz, CA, USA) or anti-active caspase-3 antibody (Cell Signaling, Beverly, MA, USA). Diamino benzidine tetrahydrochloride (DAB; Vector Laboratories, Burlingame, CA, USA) was used as a chromogen for a single staining, and the sections were counterstained with hematoxylin. In the case of double staining, the signals for ABCG2 were detected with Permanent Red (DAKO), and subsequently, anti-CD138 or antiactive caspase-3 antibody was applied. The signals for CD138 or active caspase-3 were detected with DAB. As the negative control, staining was carried out without primary antibody. This study was performed in accordance with the author's institutional guidelines and was approved by institutional review boards.

\section{Quantification of mRNA Levels by Real-Time Reverse Transcription-PCR}

RNA was extracted from the RPMI8226, U266, and Skw6.4 cells using an RNeasy kit (Qiagen, Valencia, CA, USA) with DNase I treatment. Total RNA was subjected to reverse transcription by Superscript III (Invitrogen, Carlsbad, CA, USA), and the single strand cDNA was obtained. Levels of mRNA for ABCG2, glyceroaldehyde 3-phospate dehydrogenase (GAPDH), and b-glucuronidase (GUS) genes were verified using TaqMan gene expression assays (Applied Biosystems, Foster City, CA, USA) as recommended by the manufacturer. Amount of ABCG2 mRNA was normalized to that of GAPDH mRNA, and the value was shown as relative mRNA amount. At the evaluation of effect of hypoxia, the amount of ABCG2 mRNA was normalized to that of GUS mRNA, because GAPDH expression level was affected by hypoxia. ${ }^{16}$

\section{Flow Cytometry}

Expression of ABCG2 at a protein level was examined with flow cytometry. Cells were fixed with formaldehyde, treated with BD FACS permeabilizing solution 2 (BD, Franklin Lakes, NJ, USA) in some experiments, incubated with antiABCG2 antibody conjugated with FITC (clone 5D3; MBL, Nagoya, Japan) at room temperature for $30 \mathrm{~min}$, washed, and resuspended in ice-cold phosphate-buffered saline (PBS) with $2 \%$ FCS and $1 \mu \mathrm{g} / \mathrm{ml}$ propidium iodide (BD). Signals were analyzed with FACS Calibur (BD).

Analysis for side population was carried out according to the protocol of Goodell's laboratory ${ }^{4}$ with minor modifications. Briefly, cells $\left(1 \times 10^{6}\right.$ per ml $)$ were incubated at $37^{\circ} \mathrm{C}$ for $90 \mathrm{~min}$ with $5 \mu \mathrm{g} / \mathrm{ml}$ Hoechst 33342 (Sigma), either alone or in the presence of $50 \mu \mathrm{M}$ verapamil (Sigma), collected and resuspended in ice-cold phosphate-buffered saline (PBS) with $2 \%$ FCS and $2 \mu \mathrm{g} / \mathrm{ml}$ propidium iodide (BD). Side population was analyzed with fluorescence-activated cell sorting (FACS)-Vantage (BD) carrying a triple laser. The Hoechst 33342 was excited with the UV laser at $350 \mathrm{~nm}$, and fluorescence emission was measured with 424/BP44 (Hoechst blue) and 660/BP20 (Hoechst red) optical filters.

Flow cytometric efflux assay was carried out according to the methods described previously ${ }^{17}$ with minor modifications. Cells $\left(1 \times 10^{6}\right)$ were washed once in PBS, suspended in $200 \mu \mathrm{l}$ of RPMI1640 medium supplemented with $10 \%$ FCS, and incubated with $5 \mu \mathrm{M}$ mitoxantrone (Sigma) in the presence or absence of $10 \mu \mathrm{M}$ FTC for $15 \mathrm{~min}$ at $37^{\circ} \mathrm{C}$. After incubation, cells were washed once with PBS, resuspended in $200 \mu \mathrm{l}$ of mitoxantrone-free medium with or without $10 \mu \mathrm{M}$ FTC, and then incubated for $1 \mathrm{~h}$ at $37^{\circ} \mathrm{C}$ to allow maximum efflux of fluorescent compounds. Intracellular fluorescence was measured with a $488 \mathrm{~nm}$ argon laser and a $650 \mathrm{~nm}$ longpass filter in FACS Calibur. The proportion of mitoxantrone- 
excluding cells in the treatment of ER stress inducers was divided by that without ER stress inducers, as shown dye exclusion index.

\section{Demethylation, Bisulfite Modification, and DNA Sequencing Analysis}

Skw6.4 cells were treated with $20 \mathrm{ng} / \mathrm{ml}$ IL-6 for 1 week. In some experiments, $2 \mu \mathrm{M}$ of demethylating reagent 5 -aza dC was added for 3 days. Genomic DNA $(1 \mu \mathrm{g})$ extracted from the Skw6.4, RPMI8226, U266, and some paraffin sections of lymphoid tissues was modified by sodium bisulfite using EpiTect Bisulfite Kit (Qiagen). PCR was carried out with the modified DNA as a template. The PCR reaction mixture contained $1 \mu \mathrm{l}$ of DNA in a total volume of $25 \mu \mathrm{l}, 2.5 \mu \mathrm{l}$ of $10 \times$ PCR buffer II, $5 \mu \mathrm{M} \mathrm{MgCl}_{2}, 250 \mathrm{mM}$ deoxynucleoside triphosphate, $1 \mu \mathrm{M}$ of each primer, and 1.25 units Taq gold. The PCR buffer II, $\mathrm{MgCl}_{2}$, and Taq gold are parts of the AmpliTaq Gold with Gene Amp kit (Applied Biosystems). Sequence of the primers was as follows: $5^{\prime}$-TTTTTTTAGAA TTTTATTTATTGAAATTA and 5'-CAAAAACAAACCAAAC ACTCC. The PCR was carried out at $95^{\circ} \mathrm{C}$ for $10 \mathrm{~min}$, followed by 45 cycles at $95^{\circ} \mathrm{C}$ for $1 \mathrm{~min}, 55^{\circ} \mathrm{C}$ for $1 \mathrm{~min}$, and $72^{\circ} \mathrm{C}$ for $1 \mathrm{~min}$. In this condition, equal number of clones was obtained for samples with methylated and unmethylated ABCG2 promoter when equal amount of clone was mixed. Amplified PCR fragments were purifed with QIAquick gel extraction kit (Qiagen), and were subcloned into pGEM-T Easy vector (Promega, Madison, WI, USA). The sequences of the amplified fragments were analyzed using an ABI 3100 sequencer (Applied Biosystems). At least 10 clones were analyzed for each condition.

\section{Transient Transfection and Luciferase Assay}

The PCR-amplified fragments containing promoter and a part of exon1 $(-340$ to $+298,+1$ means a transcription initiation site; the numbering of nucleotide was obtained from Ensemble Human Genome Browser, Ensembl transcript ID, ENST00000237612) of ABCG2 gene were cloned into pSP-Luc vector containing firefly luciferase gene as reported previously. ${ }^{18}$ The reporter plasmid mutated at the ACACGT site was also constructed by PCR. The sequence of ABCG2 fragment was verified by ABI 3100 sequencer. After the DNA isolation, $10 \mu \mathrm{g}$ of the reporter plasmid together with $1 \mu \mathrm{g}$ of the phRL-TK containing Renilla luciferase gene (Promega) was transiently transfected into RPMI8226 cells by using electroporation (Bio-Rad Laboratories, Richmond, CA, USA). The luciferase activity was measured by Dual Luciferase Reporter Assay Kit (Promega).

\section{RT-PCR Detecting The Activation of XBP-1}

The reverse transcriptase (RT) product described above was amplified using the following XBP-1 primers: $5^{\prime}$-CCTTGT AGTTGAGAACCAGG and 5'-GGGGCTTGGTATATATGTGG. The amplified fragment was electrophoresed, and the spliced and nonspliced variants of XBP-1 were detected.

\section{Immunoblotting}

Cells were harvested, and nuclear extracts were prepared with NE-PER nuclear and cytoplasmic extraction kit (Thermo Fisher Scientific, Rockford, IL, USA). The lysates were separated on $10 \%$ SDS-polyacrylamide gels, transferred to Immobilon (Millipore, Bedford, MA, USA), and reacted with anti-HIF-1 alpha antibody (Epitomic, Burlingame, CA, USA) or anti-actin (Sigma). After washing, the blots were incubated with an appropriate peroxidase-labeled secondary antibody (MBL), and then reacted with Renaissance reagents (NEN, Boston, MA) before exposure.

\section{Chromatin Immunoprecipitation Assay}

Chromatin immunoprecipitation (ChIP) assay was carried out with chromatin immunoprecipitation assay kit (Upstate, Lake Placid, NY, USA) according to the manufacturer's instruction. Briefly, RPMI8226 cells were fixed with $1 \%$ formaldehyde for $10 \mathrm{~min}$, washed twice with ice-cold PBS, and sonicated 15 times for 10 seconds each at power setting ' 3 ' with the ultrasonicator (TOMY Seiko, Tokyo, Japan). Soluble chromatin solution was cleared by centrifugation and adjusted to $0.1 \%$ SDS, $1 \%$ Triton X-100, and $140 \mathrm{mM} \mathrm{NaCl}$. Immunoprecipitation reactions were performed overnight with salmon-sperm DNA/protein A agarose and anti-XBP1 (Santa Cruz Biotech), anti-HIF1 alpha (Upstate), or normal rabbit IgG (for negative control; Santa Cruz Biotech). The immunoprecipitates were washed, and reverse crosslinked at $65^{\circ} \mathrm{C}$ for $6 \mathrm{~h}$. DNA purified from immunoprecipitates was subjected to PCR analysis. The sequence of primers for amplifying ABCG2 promoter (-294 to -34$)$ was as follows: 5'-GTTCATTCCGTTCGATCCCGGAGG and 5'-CCGCCAG AGCTGAACGCAGT. DNA directly purified from soluble chromatin solution was used as a positive control (input).

\section{Small-Interfering RNA Transfection}

The predesigned siRNA against ABCG2 was purchased from ABI. The siRNA $(150 \mathrm{pmol})$ was transfected into $2 \times 10^{6}$ of RPMI8226 cells by using Amaxa cell line nucleofector kit (Amaxa Inc., Gaithersburg, MD, USA), and the cells were used for experiments after $48 \mathrm{~h}$. The knocked-down expression of ABCG2 gene was verified by real-time RT-PCR in RNA level and by flow cytometry in protein level; the reduction ratio was 72.5 and $45.7 \%$, respectively. Cells transfected with irrelevant oligonucleotides were used for a negative control.

\section{Proliferation Assay}

The proliferative activity of RPMI 8226 cells was assessed with WST-1 assay according to the manufacturer's instructions.

\section{Statistical. Analysis}

Statistical analysis was carried out using Student's $t$-tests. The values are shown as the mean \pm s.e. of at least three experiments. 


\section{RESULTS}

The expression of ABCG2 in lymph nodes and tonsils was examined with immunohistochemical analysis. Strong signals for ABCG2 were detected in a limited number of cells mainly located in the interfollicular space of tonsil with lymphoid follicular hyperplasia (Figure 1a, hereafter called ABCG2high cells). The ABCG2-high cells possessed ample cytoplasm and condensed chromatin structures in the nucleus (Figure 1a, inset), the features consistent with plasma cells. The ABCG2-high cells were positive for plasma cell marker CD138 (Figure 1b), and were negative for CD20, CD79a, CD3, c-kit, and fascin (data not shown). These findings indicated that ABCG2-high cells were a small population of plasma cells. Although some plasma cells were slightly stained with ABCG2 (Figure 1a, inset), the intensity of signals of ABCG2-high cells was significantly stronger than that of other cells.

The signals for ABCG2 protein seemed to be constantly detected mainly in the cytoplasm of plasma cells (Figure 1a and b). To examine whether the ABCG2 protein was present in the cell surface, the flow cytometry was carried out using a monoclonal antibody detecting the extracellular region of
ABCG2. When RPMI8226 plasma cells under a nonpermeabilizing condition were stained, the signal for ABCG2 was detected (Figure 1c), indicating that ABCG2 was located in the surface of plasma cells. In a permeabilizing condition, ABCG2 were detected in both the cell surface and the cytoplasm, with increased the staining intensity in the cytoplasm as compared to that of a nonpermeabilizing condition. These results indicated that the ABCG2 was located not only in the cytoplasm but also in the cell surface of plasma cells.

Further immunohistochemical analysis for ABCG2 was carried out on lymphoid tissues of various pathological conditions. Cases, in which at least one ABCG2-high plasma cell was detected in 10000 lymphoid cells, were considered as ABCG2-high plasma cell ${ }^{+}$. In cases with malignant lymphomas, only nonneoplastic small lymphoid cells were counted. ABCG2-high plasma cells were detected in all of the cases with reactive lymphoid hyperplasia (Figure 1d). Among lymphomas, percentage of ABCG2-high plasma cell ${ }^{+}$cases was low in DLBCL $(5 \%)$ and FL (11\%) but high in HL (60\%), PTCL-u (42\%), and AILT (52\%) (Figure 1d).
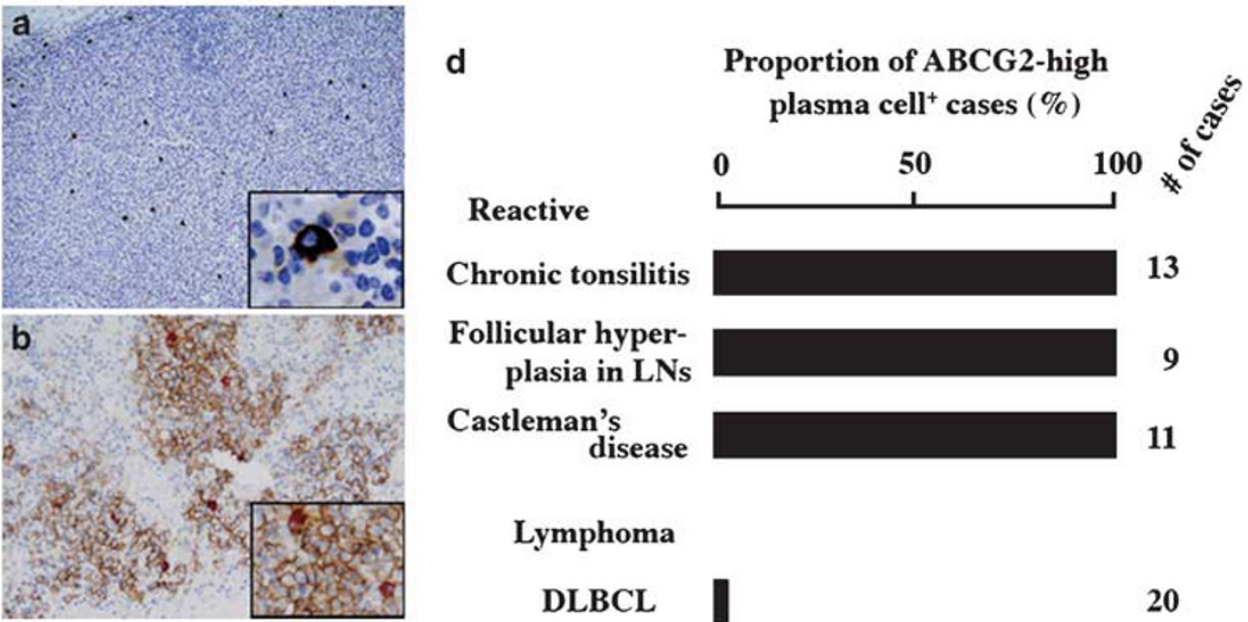

Reactive

Chronic tonsilitis

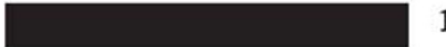

\section{Follicular hyper- plasia in LNs \\ Castleman's}

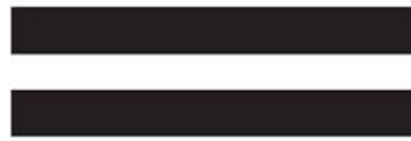
disease

\section{Lymphoma}
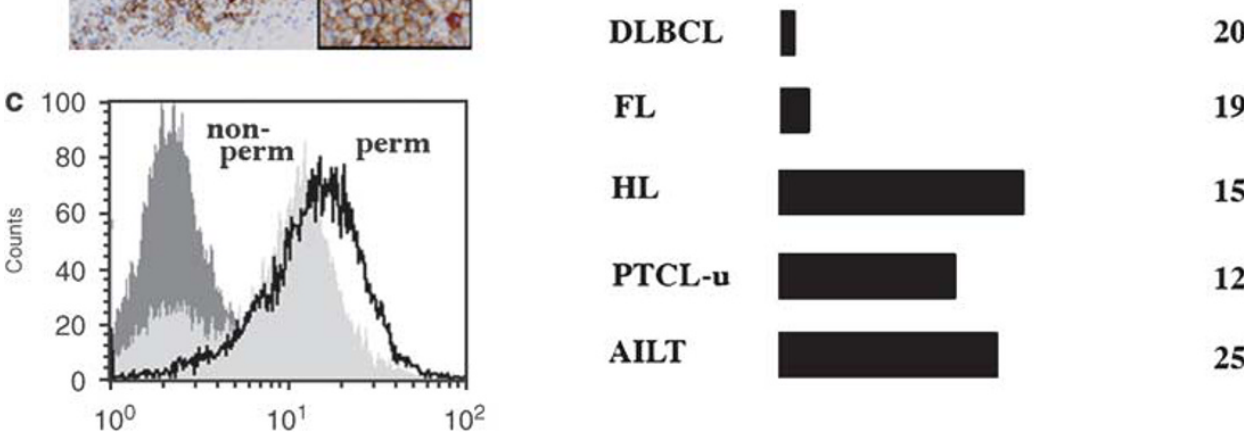

15

12

25

Figure 1 Expression of ABCG2 gene in lymph nodes and tonsils. (a) Immunohistochemical analysis of ABCG2 in tonsils affected by chronic tonsillitis. High level of expression was detected in a small population of cells located at interfollicular spaces. Strongly stained cells possessed rich cytoplasm and condensed chromatin structures in the nucleus (inset). Magnification $\times 20$ (inset, $\times 400$ ). (b) Double immunostaining with anti-ABCG2 and anti-CD138 antibodies. The signals for ABCG2 were detected as red, and those for CD138 as brown color. Magnification $\times 100$ (inset, $\times 200$ ). (c) Flow cytometric evaluation of ABCG2 expression in the nonpermeabilizing and the permeabilizing conditions. The black line showed the ABCG2 protein signal in RPMI8226 cells in the permeabilizing condition. The thin-shaded area showed the ABCG2 protein signal in the nonpermeabilizing condition. The thick-shaded area showed the signal for secondary antibody alone. (d) Prevalence of ABCG2-high plasma cells in lymph nodes and tonsils with various pathological conditions. Proportion of ABCG2-high plasma cell ${ }^{+}$cases was shown. DLBCL: diffuse large B-cell lymphoma, FL: follicular lymphoma, HL: Hodgkin lymphoma, PTCL-u: peripheral T-cell lymphoma, unspecified, AILT: angioimmunoblastic T-cell lymphoma. 
HL, PTCL-u, and AILT lesions are known to be cytokine rich as compared to FL and DLBCL. ${ }^{19-22}$ In Castleman's disease of plasma cell type, varying amount of plasma cells is present in the interfollicular area. Because cytokines might be also abundantly produced in chronic tonsillitis lesions, increase of ABCG2-high plasma cells might be attributable to cytokine-rich situation. IL-6 is the most important cytokine for differentiation of B lymphocytes to plasma cells. ${ }^{23}$ Plasma cells are subjected to ER stress when excess proteins (immunoglobulins) are synthesized. ${ }^{14}$ From these findings, it is hypothesized that ABCG2 expression might be enhanced in the plasma cells that are subjected to ER stress under IL-6 stimulation. As isolation of plasma cells from human peripheral blood is difficult, we utilized plasma cell lines hereafter.

First, the effect of IL-6 and ER stress inducer, tunicamycin, on the level of ABCG2 mRNA was examined in RPMI8226 plasma cells. In the absence of IL-6, the ABCG2 expression level increased when 10 and $20 \mu \mathrm{g} / \mathrm{ml}$ tunicamycin were added (Figure 2a). By contrast, in the presence of IL-6, even $5 \mu \mathrm{g} / \mathrm{ml}$ of tunicamycin significantly increased ABCG2 expression level (Figure 2a). Flow cytometry analysis on RPMI8226 cells in nonpermeabilizing condition revealed that the amount of cell surface ABCG2 protein increased when IL-6 or tunicamycin was added (Figure 2b, upper and middle, respectively). The cell surface ABCG2 protein level further increased when both IL- 6 and tunicamycin were added (Figure 2b, lower).

The increase of ABCG2 expression level by other ER stress inducers, thapsigargin and 2-deoxyglucose, was examined in the presence or absence of IL-6. Without IL-6, any significant increase was not observed with administration of thapsigargin or 2-deoxyglucose. In contrast, the increase of ABCG2 expression was observed by coadministration of thapsigargin or 2-deoxyglucose and IL-6 (Figure 2c).

The data on RPMI8226 plasma cells indicated that the ABCG2 expression level increased under ER stress and its level became higher in the presence of IL-6. To examine whether this was applicable to another plasma cell line or not, the expression level of ABCG2 mRNA was determined in U266 plasma cells. When tunicamycin was added, ABCG2 expression level increased 4 times and 8 times in the absence and presence of IL-6, respectively (Figure $2 \mathrm{~d}$ ). By adding thapsigargin, ABCG2 expression level increased 1.5 times and 3 times in the absence and presence of IL-6, respectively (Figure 2d).

To examine whether the cell surface ABCG2 protein induced by ER stressor and IL-6 was functional or not, the proportion of side population representing the functional ABCG2 was determined. In the presence of IL- 6 and/or tunicamycin, the proportion of side population increased in both RPMI8226 and U266 cells (Figure 2e). Furthermore, the function of ABCG2 was examined using dye exclusion assay. Both RPMI8226 and U266 showed the increase of proportion of dye-excluding cells when ER stressors, such as tunicamy- cin, thapsigargin, and 2-deoxyglucose, were added in the presence of IL-6 (Figure 2f).

Skw6.4 is a human B lymphoblastoid cell line which could differentiate to plasma cells by stimulation with IL- 6 for 1 week. ${ }^{24}$ The differentiated Skw6.4 cells did not express ABCG2 at detectable level even by treatment with IL-6 and tunicamycin (Figure 3a). Recent report showed that ABCG2 expression is regulated by epigenetic mechanism, ${ }^{25}$ suggesting a possibility that the methylation of $\mathrm{CpG}$ sequences in the promoter region may affect the expression of ABCG2 in Skw6.4. The results showed that the $\mathrm{CpG}$ sequences in the ABCG2 promoter region were heavily methylated in the differentiated Skw6.4 (Figure 3b). When the proportion of methylated $\mathrm{CpG}$ was reduced with demethylating reagent 5aza dC (Figure 3b), the ABCG2 expression increased in the differentiated Skw6.4. Its expression level increased by adding tunicamycin, and further increased by adding IL-6. The highest expression level was observed when both IL-6 and tunicamycin were added (Figure $3 a$ ).

The effect of IL-6 and ER stress inducers was more remarkable in U266 than in RPMI8226 (Figures 2a and d), suggesting a possibility that the promoter region of ABCG2 gene might be more methylated in RPMI8226 than in U266, thereby blunting the effect of IL-6 and ER stress inducers on RPMI8226 cells. To examine this, the proportion of methylated CpG sequences was compared between RPMI8226 and U266. Whereas most CpG sequences were unmethylated in U266, approximately half of CpG was methylated in RPMI8226 (Figure 3c).

It is well known that tunicamycin enhances transcription of ER stress-responsive genes through activation of transcription factor XBP-1. ${ }^{26}$ IL-6 is known to activate hypoxia-related transcription factor HIF-1. ${ }^{27,28}$ Very recently, tunicamycin has been reported to enhance HIF-1 transcription. ${ }^{29}$ To examine whether these were applicable to RPMI8226 cells, the spliced XBP-1 mRNA indicating activation of XBP- 1 was detected by RT-PCR. In the presence of tunicamycin, the amount of spliced XBP-1 mRNA increased (Figure 4a, arrowhead). Immunoblotting results revealed that the amount of HIF-1 increased slightly in the presence of IL-6, and obviously in the presence of tunicamycin or both (Figure $4 \mathrm{~b}$ ). ChIP assay reveled that XBP- 1 was recruited to the promoter region of ABCG2 gene in the presence of tunicamycin, and the amount of recruited XBP-1 increased by the addition of IL-6 (Figure 4c). The amount of HIF-1 that was recruited to the ABCG2 promoter also increased in the presence of IL-6 (Figure 4c), indicating that the recruited amount of XBP-1 and HIF-1 reached maximum when both IL- 6 and tunicamycin were added.

In the promoter region of ABCG2 gene, the sequence ACACGT was located between -172 and -167 , at which the binding sequence of XBP-1 (ACACG) and that of HIF-1 (CACGT) overlapped. To examine the role of the ACACGT site for transcription of ABCG2, following luciferase reporter plasmids were constructed; 1) the plasmid containing the 
a

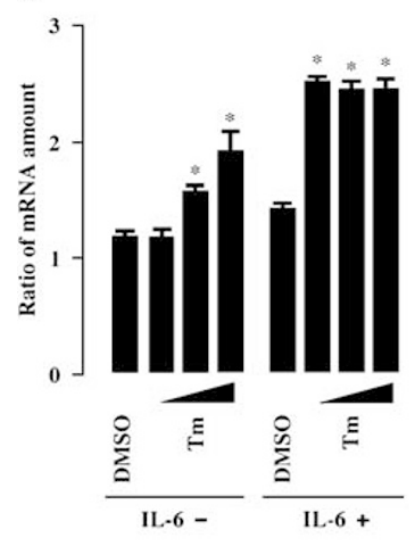

e
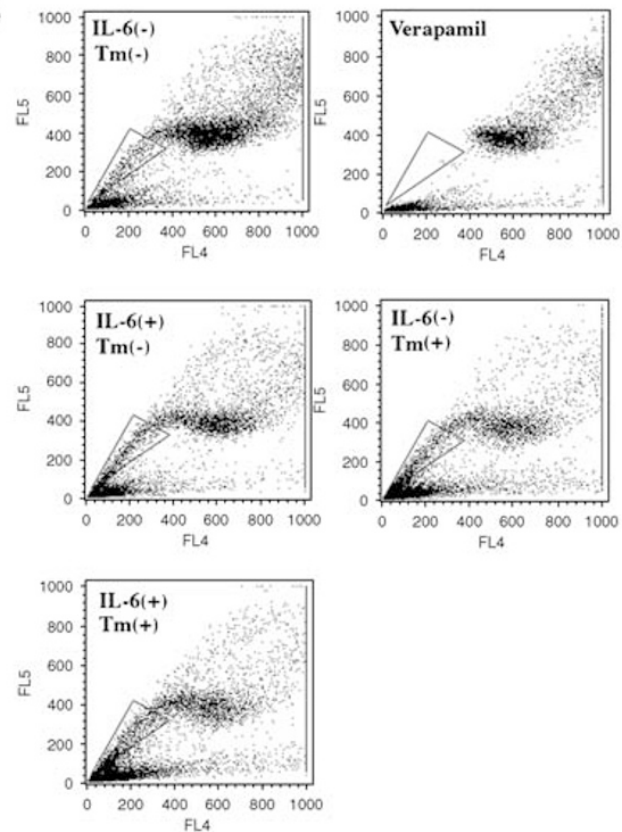

b
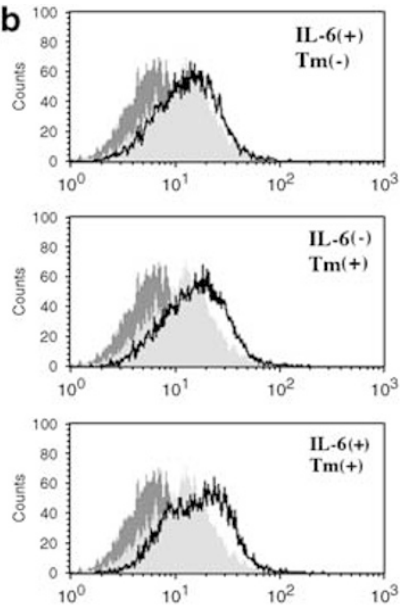
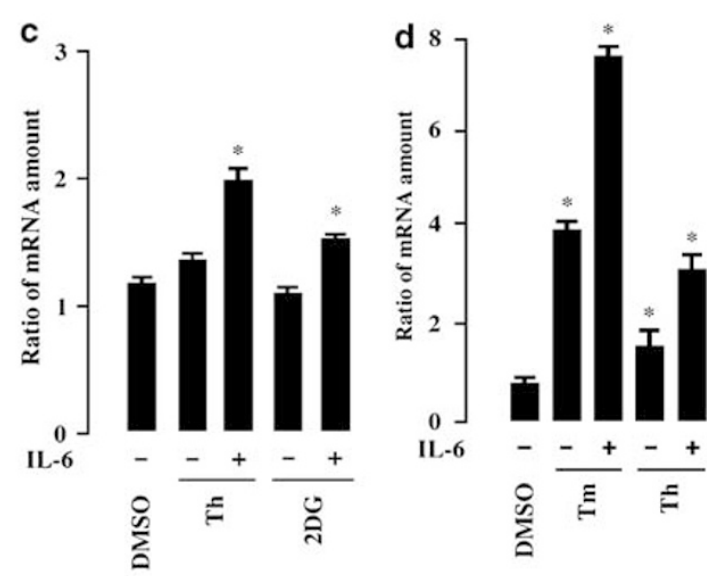
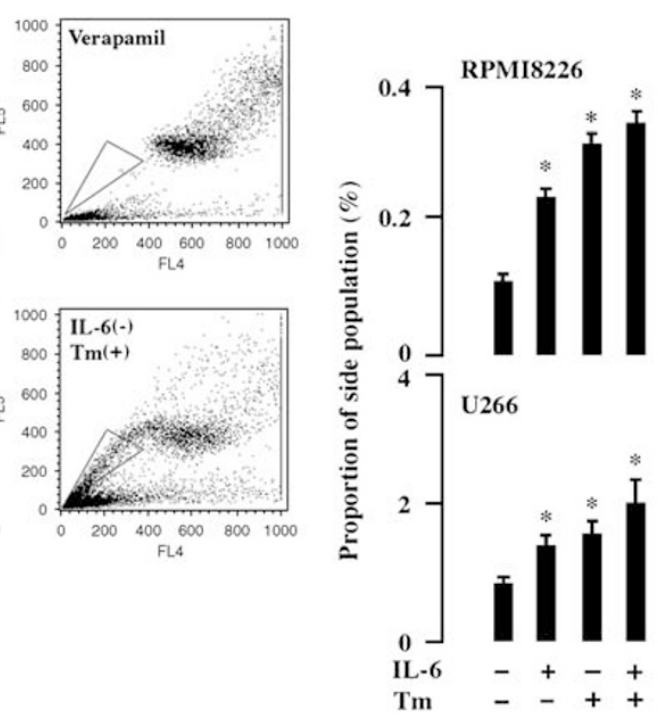

\section{f}
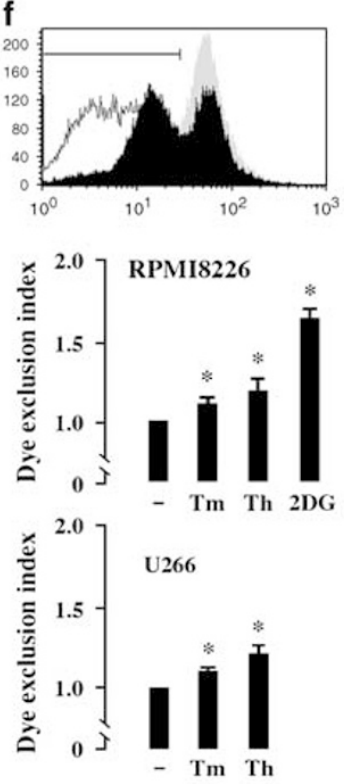

Figure 2 Effect of IL- 6 and ER stress inducers on theABCG2 expression in RPMI8226 and U266 plasma cells. (a) Real-time RT-PCR. Expression level of ABCG2 mRNA was examined in RPMI8226 cells treated with $0,5,10$, or $20 \mu \mathrm{g} / \mathrm{ml}$ tunicamycin (shown as Tm) for $24 \mathrm{~h}$ in the presence or absence of $20 \mathrm{ng} / \mathrm{ml} \mathrm{IL}-6$. ${ }^{*} P<0.01$ by Student's $t$-test versus the value of cells treated with DMSO. (b) Flow cytometric evaluation of ABCG2 expression in the presence or absence of $20 \mathrm{ng} / \mathrm{ml} \mathrm{IL-6}$ and $10 \mu \mathrm{g} / \mathrm{ml}$ tunicamycin under the non-permeabilizing condition. The black line showed the ABCG2 protein signal in RPMI8226 cells treated with IL-6 alone (upper), tunicamycin alone (middle), and both (lower), respectively. The thin-shaded area showed the signal for ABCG2 in RPMI8226 cells without any treatment, and the thick-shaded area showed the signal for secondary antibody alone. (c) Expression level of ABCG2 mRNA was examined by real-time RT-PCR in RPMI8226 cells incubated with $1 \mu \mathrm{M}$ thapsigargin (shown as Th) or $20 \mathrm{mM}$ 2-deoxyglucose (shown as $2 \mathrm{DG}$ ) for $24 \mathrm{~h}$ in the presence or absence of $20 \mathrm{ng} / \mathrm{ml} \mathrm{IL-6.} \mathrm{(d)} \mathrm{Effect} \mathrm{of} \mathrm{IL-6} \mathrm{and} \mathrm{ER} \mathrm{stress} \mathrm{inducers} \mathrm{on} \mathrm{U266} \mathrm{plasma} \mathrm{cells.} \mathrm{Expression} \mathrm{level} \mathrm{of} \mathrm{ABCG2} \mathrm{mRNA} \mathrm{was} \mathrm{examined} \mathrm{by} \mathrm{real-time} \mathrm{RT-PCR}$ in U226 cells incubated with $5 \mu \mathrm{g} / \mathrm{ml}$ tunicamycin or $1 \mu \mathrm{M}$ thapsigargin for $24 \mathrm{~h}$ in the presence or absence of $20 \mathrm{ng} / \mathrm{ml} \mathrm{IL-6.} \mathrm{(e)} \mathrm{Side} \mathrm{population} \mathrm{detected} \mathrm{in}$ cells with the treatment of IL- 6 and ER stress inducers. Typical dot blot analysis was presented in the left, and the proportion of side population was shown in the right. ${ }^{*} P<0.01$ for (c) and (d) by Student's $t$-test versus the value without IL- 6 and ER stress inducers. (f) Dye exclusion analysis. Typical histogram was shown in the upper. The thin- and thick-shaded area showed the signal for cells treated with mitoxantrone in the presence or absence of FTC, respectively. The black line showed the signal for cells without mitoxantrone treatment. The increase of dye excluding cell population with the treatment of various ER stress inducers was shown in the middle (RPMI8226) and the lower (U266). ${ }^{*} P<0.01$ by Student's $t$-test versus the value without ER stress inducers.

promoter and a part of exon 1 of ABCG2 gene ( -340 to +298 ), in which the ACACGT site was intact, and 2) the plasmid with the same length of ABCG2 sequence with mutation at the ACACGT site (ACACGT to TGTGCA). When the reporter plasmid with intact ACACGT site was transfected to RPMI8226 plasma cells, the luciferase activity increased by the addition of either IL- 6 or tunicamycin, and further increased by the addition of both IL- 6 and tunicamycin (Figure 4d). In contrast, the luciferase activity remained low in any treatments when the reporter plasmid with mutation at ACACGT site was transfected (Figure 4d). 

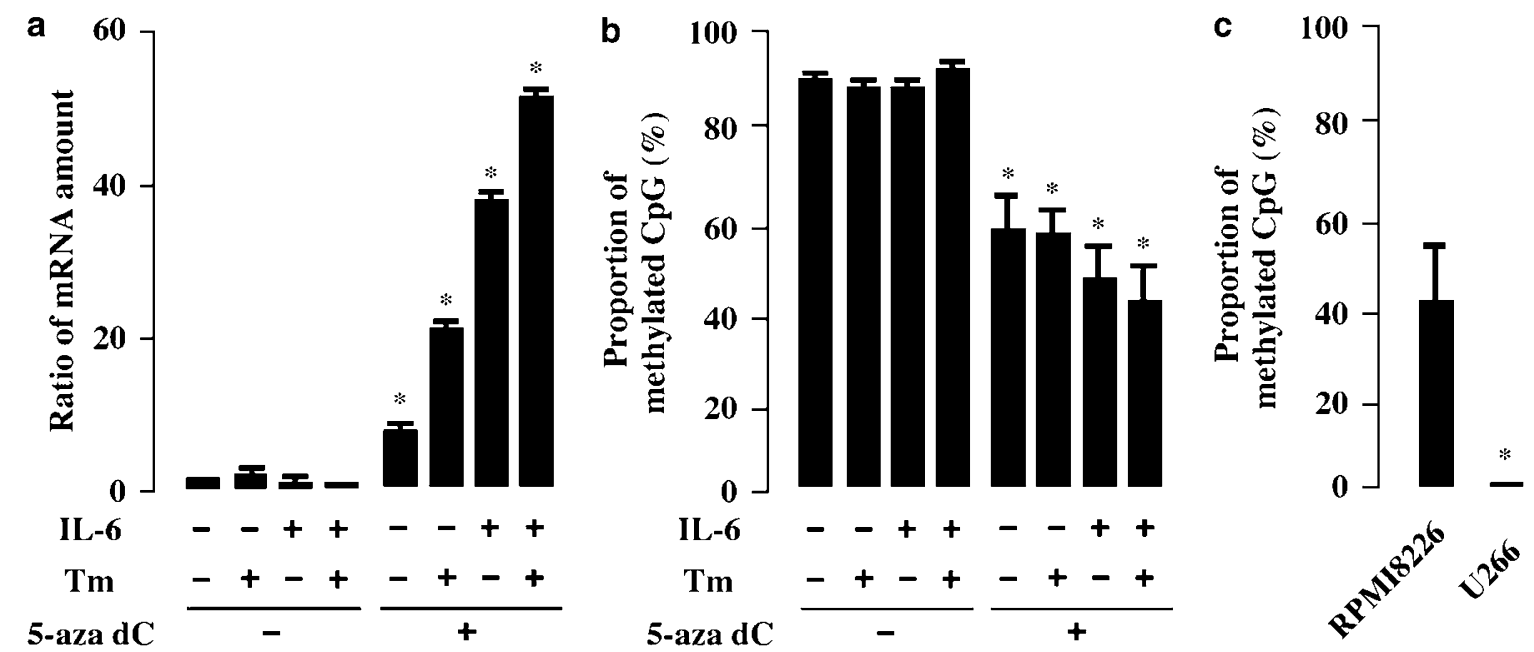

Figure 3 Effect of promoter methylation on the expression of ABCG2. (a) Expression level of ABCG2 mRNA was examined by real-time RT-PCR in Skw6.4 cells incubated with $20 \mathrm{ng} / \mathrm{ml} \mathrm{IL-6}$ for 1 week. After washing, the cells were treated with either $10 \mu \mathrm{g} / \mathrm{ml}$ tunicamycin or $20 \mathrm{ng} / \mathrm{ml} \mathrm{IL}-6$, or with both of them for $24 \mathrm{~h}$ (left). In another experiment, the cells were incubated with $2 \mu \mathrm{M}$ 5-aza dC for 3 days, and then were treated with tunicamycin and/or IL-6 (right). ${ }^{*} P<0.01$ by Student's $t$-test versus the value without IL-6, tunicamycin, and 5 -aza dC treatment. (b) Proportion of methylated CpG sequences in the promoter region of ABCG2 gene. Genomic DNA extracted from cells was modified by sodium bisulfite, and determined the proportion of methylated CpG sequences. ${ }^{*} P<0.01$ by Student's $t$-test versus the proportion in cells without any IL-6, tunicamycin, and 5-aza dC treatment. (c) Proportion of methylated CpG sequences in the promoter region of ABCG2 gene in RPMI8226 and U266. ${ }^{\star} P<0.01$ by Student's $t$-test versus U266.

ABCG2 is reported to be involved in protecting cells from apoptosis, ${ }^{11}$ and ER stress is known to induce apoptosis. ${ }^{30}$ The effect of ABCG2 on escape of cells from apoptosis was investigated. Function of ABCG2 was inhibited in RPMI8226 plasma cells transfected with specific small-interfering (si) RNA oligonucleotides, or treated with a known ABCG2 inhibitor FTC. After adding $5 \mu \mathrm{g} / \mathrm{ml}$ tunicamycin, the viability of ABCG2 knocked-down cells was low as compared to that of negative control cells (Figure 5a). The FTC treatment also reduced the viability of RPMI8226 cells (Figure 5a). The comparable results were obtained in different concentration of tunicamycin ( $10 \mu \mathrm{g} / \mathrm{ml}$; Figure $5 \mathrm{a})$ and different kind of ER stress inducer (2-deoxyglucose; Figure 5b).

Apoptosis of plasma cells in lymph nodes was immunohistochemically examined with antibody for active caspase-3 (Figure 5c). The percentage of active caspase-3positive plasma cells was significantly lower in ABCG2-high plasma cells than that in ABCG2-low plasma cells (Figure 5d).

\section{DISCUSSION}

ABCG2 is a transporter that is highly expressed in a primitive subpopulation of cells, such as hematopoietic stem cells and CD7-positive lymphoid progenitor cells. ${ }^{5,7-9}$ To our knowledge, results of immunohistochemical detection of the expression of ABCG2 in lymphoid tissues have not been reported. The present study showed the strong signals for ABCG2 in a small population of CD138-positive cells with ample cytoplasm and condensed chromatin structures in the nucleus. These features indicated that the ABCG2-high cells were a limited number of plasma cells. Raaijmakers et $a l^{31}$ and Turner $e t a l^{25}$ reported the expression of ABCG2 in plasma cells with flow cytometry. In consistent with these reports, the staining for ABCG2 was detected in almost all plasma cells, but intense in a limited number of plasma cells, ABCG2-high cells.

Immunohistochemical analysis on the lymphoid tissues of various pathological conditions revealed that ABCG2-high plasma cells were commonly detected in the interfollicular space of reactive lymphoid hyperplasia and Castleman's disease. Among lymphomas, the number of ABCG2-high plasma cells was higher in HL, PTCL-u and AILT than that in DLBCL and FL. The former diseases are known to be rich in cytokines in the lesional tissues as compared to the latter diseases. $^{8,19,21,22}$ In the reactive lymphoid hyperplasia, cytokines are also abundantly produced. ${ }^{19}$ These findings indicated the connection of the number of ABCG2-high plasma cells with cytokine contents in the lesional tissues.

IL-6 is known to be the most important cytokine for plasma cells, which stimulates the production of immunoglobulins. ${ }^{23}$ As plasma cells are subjected to ER stress when excess amount of proteins are synthesized, ${ }^{14}$ there is a possibility that ABCG2 expression might be enhanced in the plasma cells that are subjected to ER stress under IL-6-rich situation. In fact, IL-6 and ER stress inducers, tunicamycin, thapsigargin and 2-deoxyglucose, synergistically increased the level of ABCG2 expression in RPMI8226 and U266 plasma cells. These findings indicated that expression level of ABCG2 is high in plasma cells under excess ER stress.

Maliepaard et $a l^{13}$ reported ABCG2 was localized to the surface of epithelial cells in colon and choroid plexus. We also found that the signals for ABCG2 were located in the surface of the epithelial cells (data not shown). In the lymphoid 

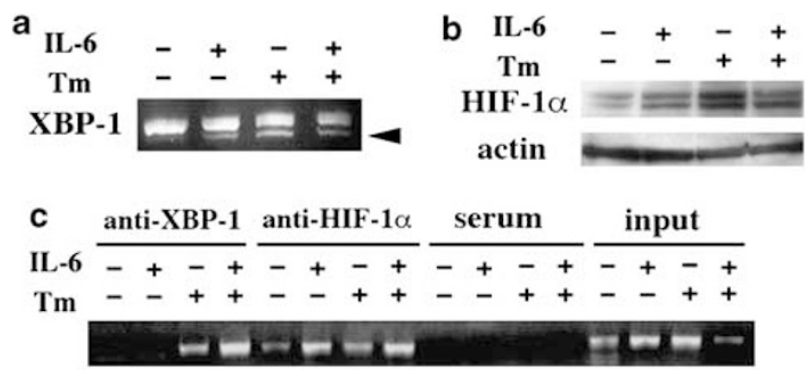

d
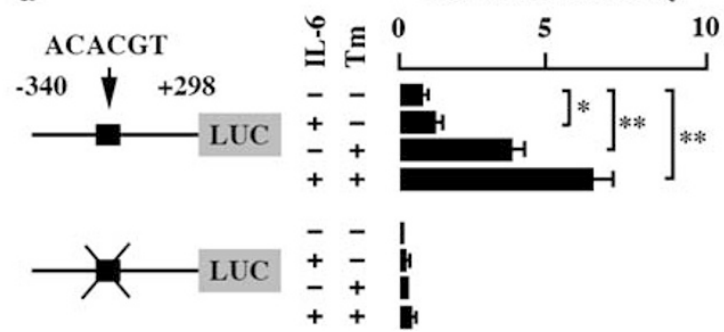

Figure 4 Effect of IL- 6 and tunicamycin on the promoter activity of ABCG2 gene. (a) Activation of XBP-1 was determined with RT-PCR. The amount of spliced form of XBP-1, indicating the activation of XBP-1, increased in the presence of tunicamycin. Arrowheads show the spliced XBP-1 form.

(b) Immunoblot showing HIF-1 $\alpha$ amount increased slightly in the presence of IL-6 and obviously in the presence of tunicamycin or in the presence of both IL-6 and tunicamycin. (c) ChIP assay. XBP-1 was recruited to the ABCG2 promoter in the presence of tunicamycin, and its recruited amount increased by the addition of IL- 6 . The recruited amount of HIF- 1 increased in the presence of IL-6. DNA directly purified from sonicated chromatin was used for positive control (input). (d) Luciferase reporter assay. The binding site for HIF-1 and XBP-1 overlapped at the ACACGT sequence. When the ACACGT site was intact, the promoter activity increased by the addition of either IL- 6 or tunicamycin, and further increased by the addition of both. No increase of promoter activity by IL- 6 and/or tunicamycin was detected when the ACACGT site was mutated. ${ }^{\star} P<0.05$ and ${ }^{\star *} P<0.01$ by Student's $t$-test versus the value without IL- 6 and tunicamycin treatment.

tissues, however, the signals for ABCG2 protein were detected mainly in the cytoplasm of plasma cells. To examine whether the ABCG2 protein was present in the cell surface, the flow cytometry was carried out using a monoclonal antibody detecting the extracellular region of ABCG2. Flow cytometry in a nonpermeabilizing condition showed the presence of ABCG2 on the cell surface of plasma cells. In addition, the increase of signal intensity with flow cytometry in a permeabilizing condition revealed the presence of ABCG2 in the cytoplasm as well. These results indicated that the ABCG2 was located not only in the cytoplasm but also in the cell surface of plasma cells, which was consistent with the results obtained by immunohistochemistry. Whether ABCG2 on cell surface is functional or not was evaluated by dye-excluding ability. ${ }^{4,17}$ The proportion of side population and the number of dye-excluding cells increased when IL-6 and ER stress inducers were added, indicating that the ABCG2 induced by IL-6 and ER stressor appeared to be functional.

Turner $e t a l^{25}$ reported that the methylation status of CpG sequences in the promoter region regulated the expression of ABCG2 gene. This was consistent with the present results that the differentiated Skw6.4 cells expressed ABCG2 only when the proportion of methylated CpG sequences in the promoter region reduced with treatment of demethylating reagent. It is well known that the chromatin structure becomes open to access for transcription factors by demethylation. ${ }^{32}$ When the demethylating reagents are added to the differentiated Skw6.4 cells, some transcription factors mediating ABCG2 expression may access to the promoter region.

The methylation status of ABCG2 promoter was further examined in clinical samples of various lymphoid tissues. However, no obvious difference was detected between tissues with numerous ABCG2-high plasma cells and those with few ABCG2-high plasma cells (data not shown). This might be due to the interference of DNA derived from nonplasma cells present in the tissues. Further analysis including single-cell microdissection would be needed to examine methylation status of plasma cells in clinical samples.

The present luciferase reporter assays revealed that the ACACGT site appeared to be essential for ABCG2 trasncription. When ACACGT site was intact, the reporter activity increased when either IL- 6 or tunicamycin was added, and further increased when both of them were added. In contrast, the reporter activity did not change with the treatment of IL- 6 and tunicamycin when the ACACGT site was mutated. The binding sites of XBP-1 and HIF-1 overlap at the ACACGT site, and XBP-1 is known to be activated by ER stress. ${ }^{26}$ IL-6, as well as ER stress inducers, increases HIF-1 expression level. ${ }^{27-29}$ IL-6 and ER stress inducers may enhance transcription of ABCG2 synergistically through the binding of XBP-1 and HIF-1 to ACACGT site.

Recently, Evseenko et $a l^{11}$ reported that ABCG2 is a placental survival factor. When ABCG2 expression level reduces, idiopathic human fetal growth restriction occurs. The knocked-down of ABCG2 expression by siRNA and the addition of ABCG2 inhibitor to trophoblast cell line induced a vulnerability of the cells to the apoptotic stimuli. In the present study, the effect of ABCG2 on the survival of plasma cells was examined using two ER stress inducers: tunicamycin and 2-deoxyglucose. The cell viability was reduced in the knocked-down of ABCG2 or the addition of FTC, indicating that ABCG2 played a role for cell survival. Although there is no evidence showing that the tunicamycin and 2-deoxyglucose are the substrate of ABCG2, it is possible that these reagents are transported by ABCG2, thereby protecting cells from apoptosis. ABCG2 may be involved in protecting not only trophoblasts but also plasma cells from apoptosis.

Taken together, a small population of plasma cells expresses a high level of ABCG2. The high expression of ABCG2 appeared to be induced by the synergistic action of IL-6 and ER stress, and could protect the plasma cells from apoptotic stimuli. 
a

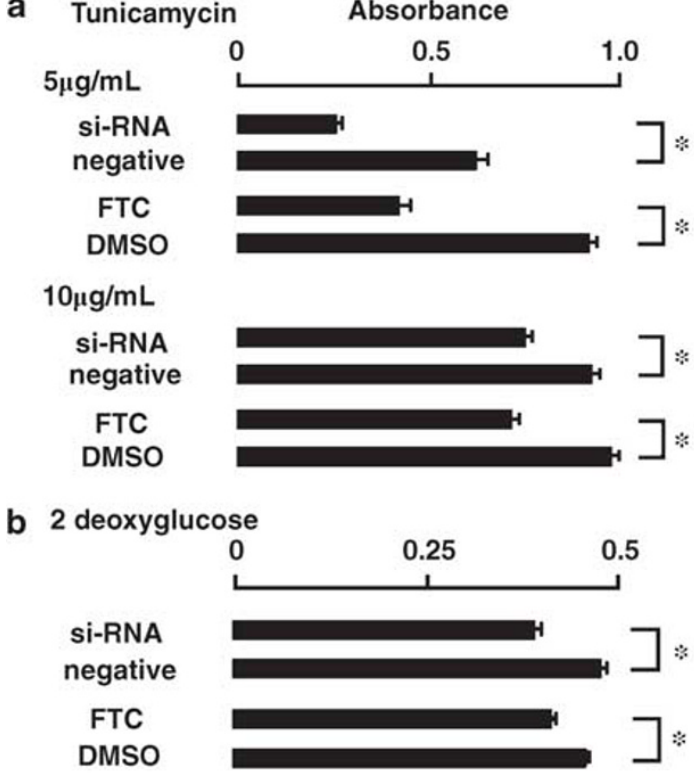

C
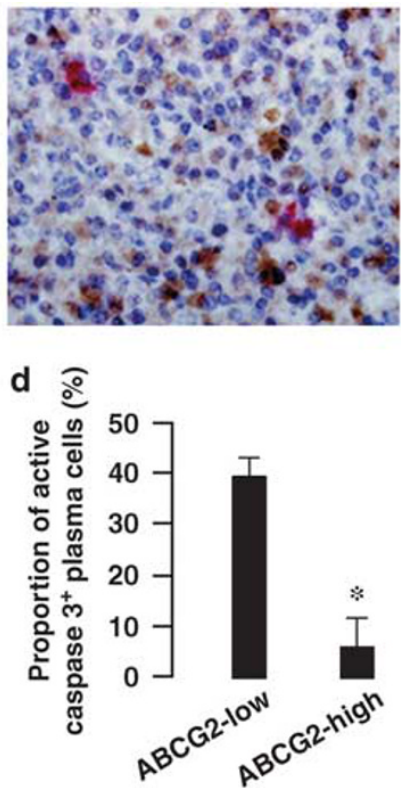

Figure 5 Effect of ABCG2 inhibition in plasma cells. (a) ABCG2 expression was knocked-down by siRNA oligonucleotides in RPMI8226 cells, and their proliferative activity was compared to that of negative control cells in the presence of tunicamycin. In another experiment, RPMI8226 cells were incubated with $10 \mu \mathrm{M} \mathrm{ABCG} 2$ inhibitor FTC for $48 \mathrm{~h}$, and the effect on the proliferative activity was determined in the presence of tunicamycin. Two concentrations ( 5 and $10 \mu \mathrm{g} / \mathrm{ml}$ ) were examined. (b) Effect of ABCG2 knocked-down and FTC was determined in the presence of $20 \mathrm{mM} 2$-deoxyglucose. ${ }^{\star} P<0.01$ by Student's $t$-test versus the value of negative control. (c) Double immunostaining with anti-ABCG2 and antiactive caspase-3 antibodies. The signals for ABCG2 were detected as red, and those for active caspase-3 as brown color. Magnification $\times 200$. (d) Proportion of active caspase-3-positive cells was counted in ABCG2-high and in other (ABCG2-low) plasma cells. ${ }^{\star} P<0.01$ by Student's $t$-test versus the proportion of $A B C G 2$-high plasma cells.

\section{ACKNOWLEDGEMENTS}

We thank Ms Megumi Sugano, Ms Takako Sawamura, Ms Etsuko Maeno, and Mr Masaharu Kohara for their technical assistant. This work was supported by grants from the Ministry of Education, Culture, Sports, Science and Technology.

1. Dean M, Rzhetsky A, Allikmets R. The human ATP-binding cassette (ABC) transporter superfamily. Genome Res 2001;11:1156-1166.

2. Hollenstein K, Dawson RJ, Locher KP. Structure and mechanism of $A B C$ transporter proteins. Curr Opin Struct Biol 2007:17:412-418.

3. Doyle LA, Ross DD. Multidrug resistance mediated by the breast cancer resistance protein BCRP (ABCG2). Oncogene 2003;22:7340-7358.

4. Goodell MA, Rosenzweig $M, \mathrm{Kim} \mathrm{H}$, et al. Dye efflux studies suggest that hematopoietic stem cells expressing low or undetectable levels of CD34 antigen exist in multiple species. Nat Med 1997;3:1337-1345.

5. Scharenberg CW, Harkey MA, Torok-Storb B. The ABCG2 transporter is an efficient Hoechst 33342 efflux pump and is preferentially expressed by immature human hematopoietic progenitors. Blood 2002;99: 507-512.

6. Zhou S, Schuetz JD, Bunting KD, et al. The ABC transporter Bcrp1/ ABCG2 is expressed in a wide variety of stem cells and is a molecular determinant of the side-population phenotype. Nat Med 2001;7:10281034.

7. Abbott BL. ABCG2 (BCRP) expression in normal and malignant hematopoietic cells. Hematol Oncol 2003;21:115-130.

8. Hao QL, Zhu J, Price MA, et al. Identification of a novel, human multilymphoid progenitor in cord blood. Blood 2001;97:3683-3690.

9. Storms RW, Goodell MA, Fisher A, et al. Hoechst dye efflux reveals a novel CD7(+)CD34(-) lymphoid progenitor in human umbilical cord blood. Blood 2000;96:2125-2133.

10. Krishnamurthy $P$, Schuetz JD. Role of $A B C G 2 / B C R P$ in biology and medicine. Annu Rev Pharmacol Toxicol 2006;46:381-410.

11. Evseenko DA, Murthi P, Paxton JW, et al. The ABC transporter BCRP/ $A B C G 2$ is a placental survival factor, and its expression is reduced in idiopathic human fetal growth restriction. FASEB J 2007;21:3592-3605.
12. Fetsch PA, Abati A, Litman $T$, et al. Localization of the ABCG2 mitoxantrone resistance-associated protein in normal tissues. Cancer Lett 2006;235:84-92.

13. Maliepaard M, Scheffer GL, Faneyte IF, et al. Subcellular localization and distribution of the breast cancer resistance protein transporter in normal human tissues. Cancer Res 2001;61:3458-3464.

14. Cenci S, Sitia R. Managing and exploiting stress in the antibody factory. FEBS Lett 2007;581:3652-3657.

15. Krishnamurthy $P$, Schuetz JD. The $A B C$ transporter Abcg2/Bcrp: role in hypoxia mediated survival. Biometals 2005;18:349-358.

16. Yamaji R, Fujita K, Takahashi $S$, et al. Hypoxia up-regulates glyceraldehyde-3-phosphate dehydrogenase in mouse brain capillary endothelial cells: involvement of $\mathrm{Na}+/ \mathrm{Ca} 2+$ exchanger. Biochim Biophys Acta 2003;1593:269-276.

17. Vethanayagam RR, Wang $\mathrm{H}$, Gupta $A$, et al. Functional analysis of the human variants of breast cancer resistance protein: $1206 \mathrm{~L}$, N590Y, and D620N. Drug Metab Dispos 2005;33:697-705.

18. Morii E, Oboki K. MITF is necessary for generation of prostaglandin D2 in mouse mast cells. J Biol Chem 2004;279:48923-48929.

19. Ho JW, Liang RH, Srivastava G. Preferential type 1-1 cytokine gene expressions in peripheral T-cell lymphomas. Hematol Oncol 1999;17:117-129.

20. Hsu SM, Waldron Jr JW, Hsu PL, et al. Cytokines in malignant lymphomas: review and prospective evaluation. Hum Pathol 1993;24:1040-1057.

21. Merz $\mathrm{H}$, Fliedner $\mathrm{A}$, Lehrnbecher $\mathrm{T}$, et al. Cytokine expression in B-cell non-Hodgkin lymphomas. Hematol Oncol 1990;8: 355-361.

22. Tesch $\mathrm{H}$, Feller AC, Jucker M, et al. Activation of cytokines in Hodgkin's disease. Ann Oncol 1992;3(Suppl 4):13-16.

23. Shapiro-Shelef $M$, Calame K. Regulation of plasma-cell development. Nat Rev Immunol 2005;5:230-242.

24. Goldstein H, Koerholz D, Chesky L, et al. Divergent activities of protein kinases in IL-6-induced differentiation of a human B cell line. J Immunol 1990;145:952-961. 
25. Turner JG, Gump JL, Zhang C, et al. ABCG2 expression, function, and promoter methylation in human multiple myeloma. Blood 2006;108:3881-3889.

26. Yoshida $\mathrm{H}$, Matsui T, Yamamoto $\mathrm{A}$, et al. XBP1 mRNA is induced by ATF6 and spliced by IRE1 in response to ER stress to produce a highly active transcription factor. Cell 2001;107:881-891.

27. Lang SA, Moser C, Gaumann A, et al. Targeting heat shock protein 90 in pancreatic cancer impairs insulin-like growth factor-I receptor signaling, disrupts an interleukin-6/signal-transducer and activator of transcription 3/hypoxia-inducible factor-1alpha autocrine loop, and reduces orthotopic tumor growth. Clin Cancer Res 2007;13:6459-6468.

28. Wigmore SJ, Sangster K, McNally SJ, et al. De-repression of heat shock transcription factor-1 in interleukin-6-treated hepatocytes is mediated by downregulation of glycogen synthase kinase 3beta and MAPK/ERK1. Int J Mol Med 2007;19:413-420.

29. Werno C, Zhou J, Brune B. A23187, ionomycin and thapsigargin upregulate mRNA of HIF-1alpha via endoplasmic reticulum stress rather than a rise in intracellular calcium. J Cell Physiol 2008;215: $708-714$.

30. Szegezdi E, Logue SE, Gorman AM, et al. Mediators of endoplasmic reticulum stress-induced apoptosis. EMBO Rep 2006;7:880-885.

31. Raaijmakers $\mathrm{MH}$, de Grouw EP, Heuver $\mathrm{LH}$, et al. Impaired breast cancer resistance protein mediated drug transport in plasma cells in multiple myeloma. Leuk Res 2005;29:1455-1458.

32. Feinberg AP, Tycko B. The history of cancer epigenetics. Nat Rev Cancer 2004;4:143-153. 